\title{
SEMILATTICES ON PEANO CONTINUA
}

\author{
W. WILEY WILLIAMS
}

ABSTRACT. A continuum is cell-cyclic if every cyclic element is an $n-$ cell for some integer $n$. It is shown that every cell-cyclic Peano continuum admits a topological semilattice.

By a semigroup we mean a Hausdorff topological space together with an associative multiplication. One of the oldest problems in semigroup theory is: "Given a space $X$ with topological properties $P$, does $X$ admit the structure of a semigroup having algebraic properties $Q$ ?". In the case when $Q$ is "commutative and idempotent", $X$ is said to be a semilattice, and another approach is open. If one can define on $X$ a partial order so that the operation $\wedge: X \times X \rightarrow X$ defined by $\wedge(a, b)=1 . u . b .\{a, b\}$ is continuous, then $(X, \wedge)$ is a semilattice. Knight has shown [3] that any Peano (locally connected metric) continuum admits a partial order with closed graph.

In order for a Peano continuum to be a semilattice it must be acyclic [6], however not all acyclic Peano continua admit a semilattice structure. Lawson and the author have shown [5] that any semilattice on a finite-dimensional Peano continuum which is not one-dimensional contains a two-cell. Thus the example given by Borsuk in [1] is a two-dimensional Peano continuum which does not admit a semilattice. We prove here that a Peano continuum every cyclic element of which is an $n$-cell for some integer $n$ admits a semilattice.

We shall use the cyclic element notation and results of Whyburn [7] and Kuratowski and Whyburn [4], slightly modified in the following way. In a Peano continuum $X$, we say $x$ separates $a$ and $b$ if each arc from $a$ to $b$ contains $x$, and a cyclic element $D$ separates $a$ and $b$ if each arc from $a$ to $b$ meets $D$. $E(a, b)$ denotes the set of points which separate $a$ and $b$ (including $a$ and $b$ ) and is a compact partially ordered set under the ordering $x \leq y$ iff $x$ separates $y$ and $a . C(a, b)$ denotes the cyclic chain from

Received by the editors December 20, 1973 and, in revised form, April 12, 1974. AMS (MOS) subject classifications (1970). Primary 22A15; Secondary 06A20.

Key words and phrases. Peano continuum, cyclic element, cell-cyclic, topological semilattice, retract. 
$a$ to $b$ and is $\{x$ : some arc from $a$ to $b$ contains $x\}$. Given a point $a$ and a cyclic chain $C(p, q)$, if $a \notin C C(p, q)$ there is a unique element $x$ of $C(p, q)$ such that $x$ separates each element of $C(p, q)$ from $a$. Denote $x$ by $P(C(p, q), a)$. If $a \in C(p, q)$, set $P(C(p, q), a)=a$. We will use $I$ to denote the unit interval under min multiplication.

Lemma 1 ([4, p. 70]). Let $X$ be a Peano continuum, and let $C$ be a fixed cyclic chain of $X$. The function $f_{c}: X \rightarrow C$ defined by $f_{c}(x)=P(C, x)$ is a monotone retraction mapping $X-C$ into the boundary of $C$.

Note that since a cyclic element is a cyclic chain between any pair of its points, the above holds for cyclic elements as well.

Lemma 2. Let $a$ be an element of a cyclic chain $C$ in a Peano continuum $X$, and let $\epsilon>0$. There exists $\delta>0$ such that for each $x$ in $X-C, P(C, x)$ in $B(a, \delta)-\{a\}$ (the deleted open ball about $a$ ) implies $x$ is in $B(a, \epsilon)$, and also $x$ in $B(a, \delta)$ implies $P(C, x)$ is in $B(a, \epsilon)$.

Proof. If $a$ is in the interior of $C$, choose $\delta<\epsilon$ such that $B(a, \delta) \subset C^{\circ}$. Otherwise the components of $X-C$ form a null sequence at most [7], so there are a finite number of diameter $>\epsilon / 2$. Choose a point $x_{i}$ in each, and let

$$
\delta<\min \left[\left\{d\left(a, P\left(C, x_{i}\right)\right) \mid d\left(a, P\left(C, x_{i}\right)\right)>0\right\} \cup\{\epsilon / 2\}\right] .
$$

Then if $P(C, y)$ is in $B(a, \delta)-\{a\}$, either $y \in C \cap B(a, \delta) \subset B(a, \epsilon)$ or $y$ is in a component of $X-C$ of diameter $<\epsilon / 2$. Thus

$$
d(y, a) \leq d(y, P(C, y))+d(P(C, y), a)<\epsilon / 2+\epsilon / 2,
$$

so the first implication holds. The second follows from Lemma 1.

The semilattice structure on $X$. A Peano continuum $X$ is cell-cyclic iff every true cyclic element of $X$ is an $n$-cell for some integer $n$. Let $K_{n}$ be $I^{n}$ under coordinatewise multiplication and $K_{n}^{\prime}$ be $T^{n}$ under coordinatewise multiplication, where $T$ is the subsemilattice $I \times\{0\} \cup\{0\} \times I$ of $I^{2}$. Then $K_{n}$ and $K_{n}^{\prime}$ are semilattices on the $n$-cell, with the former having its minimum element on the boundary and the latter having its in the interior. Fix an element 0 of $X$.

For each cyclic element $D$ of $X$ define a function $b_{D}$ as follows: If $P(D, 0)$ is on the boundary (interior) of $D$ let $b_{D}: K_{n}\left(K_{n}^{\prime}\right) \rightarrow D$ be a homeomorphism mapping the minimum element of $K_{n}\left(K_{n}^{\prime}\right)$ to $P(D, 0)$. 
Lemma 3. Let $X$ be a cell-cyclic Peano continuum with $a, b$, and $c$ in $X$. Suppose no cyclic element containing one of $b$ and $c$ separates the other from $a$. Then there exists a (possibly degenerate) cyclic element which separates any two of $a, b$, and $c$.

Proof. Case I. Some cyclic element containing one element separates the other two. Then this cyclic element separates any two of $a, b$, and $c$.

Case II. No cyclic element containing one of $a, b$, and $c$ separates the other two. This implies no two of $a, b$, and $c$ lie in the same cyclic element. Then $E(a, c) \cap E(a, b)$ is the intersection of two compact totally ordered sets whose orderings agree where possible, i.e. on the intersection, and hence has a largest element $d$. If $d$ separates $b$ and $c$, then $d$ is the required cyclic element. If $d$ does not, then $C(d, b) \cap C(d, c)$ is a true cyclic element $D$ of $X$ separating each of $b$ and $c$ from $a$. Moreover the maximality of $d$ in $E(a, c) \cap E(a, b)$ implies $D$ separates $b$ and $c$. This completes the proof.

Notation. Since the intersection of cyclic elements is a cyclic element, there is a smallest cyclic element which separates $0, a$, and $b$. Denote it by $D(a, b)$.

Lemma 4. Given three points $a, b$, and $c$ in $X$, and any $x \in D(a, b)$ and $y \in D(b, c), D(x, c)=D(a, y)$.

Proof. It suffices to note that both $D(x, c)$ and $D(a, y)$ are the maximum cyclic element in $C(0, a) \cap C(0, b) \cap C(0, c)$.

To define the semilattice operation on $X$, we denote by $\wedge$ the operation on whichever of $K_{n}$ or $K_{n}^{\prime}$ fits the context.

Given $a$ and $b$ in $X$, define

$$
a b=h_{D(a, b)}\left(h_{D(a, b)}^{-1}(P(D(a, b), a)) \wedge h_{D(a, b)}^{-1}(P(D(a, b), b))\right) .
$$

Main Theorem. Let $X$ be a cell-cyclic Peano continuum. Then under the above operation $X$ is a semilattice.

Proof. The operation is obviously idempotent and commutative. It follows from Lemma 4 that it is associative. The proof of continuity will be by cases. First note that the operation is continuous when restricted to any cyclic element. Fix an open connected set $U$ containing $a b$. We seek open sets $V$ and $W$ such that $a \in V, b \in W$ and $V W \subset U$.

Case I. $a, b$, and $a b$ all distinct. If $D(a, b)=\{a b\}$ then the components of $a$ and $b$ in $X-\{a b\}$ are the required $V$ and $W$. If $D(a, b)$ is a true cyclic 
element it is iseomorphic to $K_{n}$ or $K_{n}^{\prime}$. Thus there exist disjoint relatively open sets $V^{\prime}$ and $W^{\prime}$ of $D(a, b)$ containing $P(D(a, b), a)$ and $P(D(a, b), b)$, respectively, such that $V^{\prime} W^{\prime} \subset U$. Then $V=f_{D(a, b)}^{-1}\left(V^{\prime}\right)$ and $W=f_{D(a, b)}^{-1}\left(W^{\prime}\right)$ are the required sets.

Case II. $a=b=a b$. If this point is in $D^{\circ}$, we are done since $D$ is iseomorphic to $K_{n}$ or $K_{n}^{\prime}$, so suppose it is on the boundary of $D$. Choose $\epsilon>0$ such that $B(a, \epsilon) \subset U$. The components of $X-\{a\}$ form a null sequence at most, so let $C_{1}, \ldots, C_{n}$ be those that meet $X-B(a, \epsilon / 2)$. Some of these may contain a cyclic element $D_{i}$ containing $a$. For each of those that do, there exists a positive $\delta_{i}<\epsilon$ such that

$$
\left[B\left(a, \delta_{i}\right) \cap D_{i}\right]\left[B\left(a, \delta_{i}\right) \cap D_{i}\right] \subset B(a, \epsilon) \cap D_{i}
$$

since $D_{i}$ is a topological semilattice. Also by Lemma 2 , there exists a positive $\delta_{i}^{\prime} \leq \delta_{i}$ such that for $x \in C_{i}-D_{i}$ both $P\left(D_{i}, x\right)$ in $B\left(a, \delta_{i}^{\prime}\right)$ implies $x \in B\left(a, \delta_{i}\right)$ and $x \in B\left(a, \delta_{i}^{\prime}\right)$ implies $P\left(D_{i}, x\right) \in B\left(a, \delta_{i}\right)$.

Then choose $\delta_{i}^{\prime \prime} \leq \delta_{i}$ so that $x \in\left(C_{i}-D_{i}\right) \cap B\left(a, \delta_{i}^{\prime \prime}\right)$ implies $P\left(D_{i}, x\right)$ $\in B\left(a, \delta_{i}\right)$. If $y, z \in B\left(a, \delta_{i}^{\prime \prime}\right) \cap C_{i}$, either (i) $y$ and $z$ are in the same component of $C_{i}-D_{i}$, in which case the choice of $\delta_{i}^{\prime \prime}$ implies $y z \in B\left(a, \delta_{i}\right)$ since $P\left(D_{i}, y z\right)=P\left(D_{i}, y\right)=P\left(D_{i}, z\right) \in B\left(a, \delta_{i}^{\prime}\right)$, or $(\mathrm{ii}) y z=P\left(D_{i}, y\right) . P\left(D_{i}, z\right)$, which by the second implication is a product of two elements of $B\left(a, \delta_{i}\right) \cap$ $D_{i}$, and hence in $B(a, \epsilon) \cap D_{i}$. Thus

$$
\left[B\left(a, \delta_{i}^{\prime \prime}\right) \cap C_{i}\right]\left[B\left(a, \delta_{i}^{\prime \prime}\right) \cap C_{i}\right] \subset B(a, \epsilon) \cap C_{i}
$$

Now for those $C_{i}$ not containing such a $D_{i}$, each must contain a cut point $x_{i}$ of itself in $B(a, \epsilon / 2)$ such that $C\left(a, x_{i}\right) \subset B(a, \epsilon / 2)$. Again by Lemma 2 , there is a positive $\delta_{i}<\epsilon / 2$ such that for $x$ in $C_{i}-C\left(a, x_{i}\right)$, $P\left(C\left(a, x_{i}\right), x\right) \in B\left(a, \delta_{i}\right)$ implies $x \in B(a, \epsilon / 2)$ and $x \in B\left(a, \delta_{i}\right)$ implies $P\left(C\left(a, x_{i}\right), x\right) \in B(a, \epsilon / 2)$. Choose $\delta_{i}^{\prime}, 0<\delta_{i}^{\prime}<\delta_{i}$, so that $x \in B\left(a, \delta_{i}^{\prime}\right) \cap\left[C_{i}\right.$ $\left.C\left(a, x_{i}\right)\right]$. implies $P\left(C\left(a, x_{i}\right), x\right) \in B\left(a, \delta_{i}\right)^{2}$. By an argument similar to that of the preceding paragraph, one shows that

$$
\left[B\left(a, \delta_{i}^{\prime}\right) \cap C_{i}\right]\left[B\left(a, \delta_{i}^{\prime}\right) \cap C_{i}\right] \subset B(a, \epsilon) \cap C_{i}
$$

Finally by a technique like that above choose $\delta_{\circ}$ so that $y \in B\left(a, \delta_{\mathrm{o}}\right)$ and in the component of 0 in $X-\{a\}$ implies ay, $\in B(a, \epsilon)$. Let $\delta$ be the smallest of all the $\delta_{\circ}, \delta_{i}, \delta_{i}^{\prime}$, and $\delta_{i}^{\prime \prime}$. We claim that $B(a, \delta)^{2} \subset B(a, \epsilon)$. For if $y, z \in B(a, \delta)$ are in the same component of $X-\{a\}$, the above two paragraphs show that $y z \in B(a, \epsilon)$. If $y$ and $z$ are in different components of $X-\{a\}$ and neither of these components contain 0 , then $y z=a$, while if 
the component of, say, $y$ contains 0 , then $y z=y a \in B(a, \epsilon)$.

Case III. $a=a b \neq b$. This will be subdivided into three subcases:

(i) Suppose $D(a, b)=a$, so that $a$ separates $b$ from 0 , and also that $C(a, b)$ contains a true cyclic element $D$ containing $a$. Then $P(D, b) \neq a$ and $a b=a P(D, b)$, so there exist disjoint relatively open subsets $V^{\prime}$ and $W^{\prime}$ of $D$ such that $a \in V^{\prime}, P(D, b) \in W^{\prime}$, and $V^{\prime} W^{\prime} \subset U \cap D$. By a technique similar to that of Case II, choose a $\delta>0$ such that if $y$ is in $B(a, \delta)$ and the component of 0 in $X-\{a\}$ then $y a \in U$. Let $V=f_{D}^{-1}\left(V^{\prime}\right) \cap B(a, \delta)$ and $W=f_{D}^{-1}\left(W^{\prime}\right)$. Then for $y \in V$ and $z \in W$, either $y$ is in the component of 0 in $X-\{a\}$, in which case $y z=y a \in U$, or $y$ is not in the component of 0 in $X-\{a\}$, in which case $y z=P(D, y) \cdot P(D, z) \in V^{\prime} W^{\prime} \subset U$.

(ii) Suppose $D(a, b)=a$, but $C(a, b)$ contains no true cyclic element containing a. By Case II, there is an open set $W^{\prime}$ such that $W^{\prime} W^{\prime} \subset U$. Also $C(a, b)$ contains a cut point $x$ with the property that $C(a, x) \subset W^{\prime}$. Let $V$ be the component of $b$ in $X-\{x\}$, and let $W$ be $W^{\prime}$ intersected with the component of $a$ in $X-\{x\}$. For $y \in V$ and $z \in W, y z=x z \in W^{\prime} W^{\prime} \subset U$.

(iii) Suppose $D(a, b) \neq a$. Then $D(a, b)$ is a true cyclic element containing $a$ which separates any two of $a, b$, and 0 , so that $P(D(a, b), 0) \neq a$ and $P(D(a, b), b) \neq a$. Choose $V^{\prime}$ and $W^{\prime}$ disjoint relatively open subsets of $D(a, b)$ such that $P(D(a, b), b) \in W^{\prime}, a \in V^{\prime}, V^{\prime} W^{\prime} \subset U \cap D(a, b)$, but neither contains $P(D(a, b), 0)$. Then $V=f^{-1}\left(V^{\prime}\right)$ and $W=f^{-1}\left(W^{\prime}\right)$ are the required sets, for $y \in V$ and $z \in W$ imply

$$
y z=P(D(a, b), y) P(D(a, b), z) \in V^{\prime} W^{\prime} \subset U \text {. }
$$

This completes the proof of the main theorem.

Corollary. Every retract of $I^{2}$ admits a semilattice.

Proof. Borsuk [2] has characterized retracts of $I^{2}$ as locally connected continua which do not separate the plane. Whyburn [8] in turn has characterized these as those locally connected continua in the plane such that every true cyclic element is a simple closed curve with interior, i.e. a twocell. From these results the Corollary follows.

\section{BIBLIOGRAPHY}

1. K. Borsuk, Sur les retracts, Fund. Math. 17 (1931), 164.

2. Theory of retracts, Monografie Mat., Tom 44, PWN, Warsaw, 1967. MR $35 \# 7306$.

3. V. Knight, A continuous partial order for Peano continua, Pacific J. Math. 30 (1969), 141-153. MR $39 \# 7570$. 
4. C. Kuratowski and G. T. Whyburn, Sur les éléments cycliques et leurs applications, Fund. Math. 16 (1930), 305-331.

5. J. D. Lawson and W. W. Williams, Topological semilattices and their underlying spaces, Semigroup Forum 1 (1970), no. 3, 209-223. MR 42 \#3221.

6. A. D. Wallace, Acyclicity of compact connected semigroups, F und. Math. 50 (1961:/62), 99-105. MR 24 \#A2373.

7. G. T. Whyburn, Analytic topology, Amer. Math. Soc. Colloq. Publ., vol. 28, Amer. Math. Soc., Providence, R. I., 1942. MR 4, 86.

8. - Concerning the structure of the continuous curve, Amer. J. Math. 50 (1928), 167-194.

DEP ARTMENT OF MATHEMATICS, UNIVERSITY OF LOUISVILLE, LOUISVILLE, KENTUCKY 40208 